\title{
Detection of Ground Clutter for Dual-Polarization Weather Radar Using a Novel 3D Discriminant Function
}

\author{
MOHAMMAD-HosSEIN GOLBON-HAGHIGHI \\ School of Electrical and Computer Engineering, and Advanced Radar Research Center, \\ University of Oklahoma, Norman, Oklahoma \\ GUIFU ZHANG \\ School of Meteorology, and School of Electrical and Computer Engineering, and Advanced \\ Radar Research Center, University of Oklahoma, Norman, Oklahoma
}

(Manuscript received 25 August 2018, in final form 22 April 2019)

\begin{abstract}
A novel 3D discriminant function is introduced as part of a ground clutter detection algorithm for improving weather radar observations. The 3D discriminant function utilizes the phase fluctuations of the received signals for horizontal and vertical polarizations and the dual-scan cross-correlation coefficient. An optimal decision based on the 3D discriminant function is made using a simple Bayesian classifier to distinguish clutter from weather signals. For convenience of use, a multivariate Gaussian mixture model is used to represent the probability density functions of discriminant functions. The model parameters are estimated based on the maximum likelihood using the expectation-maximization (ML-EM) method. The performance improvements are demonstrated by applying the proposed detection algorithm to radar data collected by the polarimetric Norman, Oklahoma (KOUN), weather radar. This algorithm is compared to other clutter detection algorithms and the results indicate that, using the proposed detection algorithm, a better probability of detection can be achieved.
\end{abstract}

\section{Introduction}

It is still a challenging issue to detect ground clutter and mitigate its effects on weather observations. For weather radar, ground clutter is the undesired echoes from scatterers on the ground, which can significantly bias the polarimetric weather radar measurements. Ground clutter signals are typically statistically stationary in the time domain and mostly located around zero Doppler velocity in the spectral domain. Traditionally, a band-stop filter was employed to mitigate the ground clutter effects (Groginsky and Glover 1980; Cao et al. 2012). However, some power components of weather signals (around zero velocity) may fall into the stop band of the filter and cause bias estimates of spectral moments and polarimetric parameters (Siggia and Passarelli 2004; Doviak and Zrnić 2006; Zhang 2016). Hence, to avoid biases from unnecessary filtering, ground clutter locations need to be precisely identified.

Corresponding author: Mohammad-Hossein Golbon-Haghighi, golbon@ou.edu; Guifu Zhang, guzhang1@ou.edu
Meischner (2002) introduced a static clutter map method to identify clutter contaminated resolution volumes. To take the weather condition into account and improve the detection performance, Hubbert et al. (2009a,b) developed a clutter mitigation decision (CMD) algorithm, which includes three discriminant functions: the reflectivity texture, clutter phase alignment, and the number of reflectivity fluctuations (Steiner and Smith 2002), and uses them in combination for clutter detection.

Dual-polarization clutter detection algorithms are more attractive because they can take advantage of the polarization diversity. Ground clutter and weather signals have different polarimetric characteristics which can be utilized to distinguish clutter from weather signals (Gourley et al. 2007; Park et al. 2009; Rico-Ramirez and Cluckie 2008). In Li et al. (2014), the dual-polarization (DP) clutter detection algorithm is proposed based on three discriminant functions: the copolar cross-correlation coefficient $\rho_{\mathrm{hv}}$, power ratio (PR), and differential reflectivity $Z_{\mathrm{DR}}$. It is shown in Fig. 4 of Li et al. (2014) that there is an overlapped area between clutter and weather probability density functions (PDFs), for both $Z_{\mathrm{DR}}$ and $\rho_{\mathrm{hv}}$. 
Also, the peak values of weather PDF and clutter PDF are in the same range (i.e., near zero for $Z_{\mathrm{DR}}$ and near one for $\left.\rho_{\mathrm{hv}}\right)$. Furthermore, it is shown that the weather PDFs have larger peak values than clutter PDFs (for both $Z_{\mathrm{DR}}$ and $\left.\rho_{\mathrm{hv}}\right)$. Consequently, it is clear that clutter signals with near-zero $Z_{\mathrm{DR}}$ values and near-one $\rho_{\mathrm{hv}}$ values would most likely be detected as weather instead of clutter. Therefore, this can increase the detection error rate and reduce the probability of detection $P_{D}$.

Realizing the difference in correlation time between weather and clutter, Li et al. (2013) proposed the dualscan (DS) method to improve clutter detection. Taking advantages of the information used in both DP and DS methods, Golbon-Haghighi et al. (2016) developed the DPDS by using the joint 2D PDF of $\rho_{\mathrm{hv}}$ and $\rho_{12}$, as a discriminant function, which is computationally efficient to use for the classification. The phase fluctuation index is introduced in Golbon-Haghighi et al. (2018) and uses the polynomial fitting function to discriminate clutter from weather signals.

More recently, the spectral properties have been considered to introduce new discriminant functions and improve ground clutter detection algorithms (Cao et al. 2012; Zhang 2016; Li et al. 2011). In Li et al. (2013), the spectrum clutter identification (SCI) algorithm is introduced based on four discriminant functions: spectrum power distribution, spectrum phase fluctuation, power texture, and spectrum width texture. A dual-polarization spectral decomposition technique is introduced in Wen et al. (2017), to identify clutter from nonmeteorological echoes such as chaff, birds, insects, and sea clutter. A Bayesian radar-signal classifier is proposed in Falconi et al. (2016) to identify ground clutter regarding its spatial statistical features. In Warde and Torres (2014) and Torres and Warde (2014), the clutter environment analysis using adaptive processing (CLEAN-AP) algorithm is introduced to achieve a better performance for the clutter detection. Bachmann (2008) introduced the differential phase between the spectral coefficients of two spectra from the even and odd samples for clutter identification.

A novel 3D discriminant function is introduced in this paper to improve the performance of clutter detection algorithms. The $3 \mathrm{D}$ discriminant function is introduced based on the phase structure function for horizontal (h) and vertical (v) polarizations and the dual-scan crosscorrelation coefficient. It is found that the $3 \mathrm{D}$ discriminant function has a good separation between clutter and weather PDFs even for low values of the clutter to weather signal ratio (CSR). The CSR is defined as the ratio of the clutter power to the weather power, and expressed in decibels $(\mathrm{dB})$. Numerical simulations and theory show that the phase variations of signals received from the ground clutter are different from weather signals. The random location, motion, and distribution of scatterers cause the amplitude and phase of the received complex voltage to be random variables. The phase fluctuations of clutter signals are typically slower than that of the randomly distributed hydrometers. Hence, the structure function for the phase fluctuations is introduced to discriminate clutter from weather signals. Therefore, it is expected that the 3D discriminant function based on the phase structure function (PSF) can achieve a better performance gain for ground clutter detection algorithms.

The PDFs of discriminant functions may change based on weather conditions, types of clutter, antenna rotation and radar parameters, and thus, the PDFs may need to be updated based on different conditions. Also, complete knowledge about the probabilistic structure of class-conditional densities may not be available for realtime implementations. Therefore, a multivariate Gaussian mixture model (GMM) is introduced in this paper to parameterize the class-conditional densities for the proposed $3 \mathrm{D}$ discriminant function. The complexity of the problem is reduced significantly by using the GMM model. The GMM parameters are estimated based on the maximum likelihood using the expectation-maximization (ML-EM) method.

The remainder of this paper is organized as follows: In section 2, discriminant functions for the proposed clutter detection algorithm are introduced. In section 3 , the implementation procedures of the algorithm are discussed, and the performance of the algorithm is evaluated using controlled datasets collected by the Norman, Oklahoma (KOUN), polarimetric radar. Conclusions are drawn in section 4 .

\section{Discriminant functions}

A new discriminant function is introduced in this section to develop ground clutter detection algorithms. Controlled training and testing datasets have been used to estimate the PDF of discriminant functions. Training datasets were collected on the nearly pure weather and pure clutter conditions to estimate the ground truth and obtain the class-conditional densities (the PDF of discriminant functions) for the classification.

Controlled in-phase and quadrature-phase (I/Q) datasets are created by matching and combining the almost pure clutter and pure weather to find ground truth. The nearly pure weather data was obtained by collecting data at high elevation angles at the farther ranges (over $50 \mathrm{~km}$ ) from the radar location, where ground clutter can be neglected, while the nearly pure clutter data was obtained at the low elevation angles 

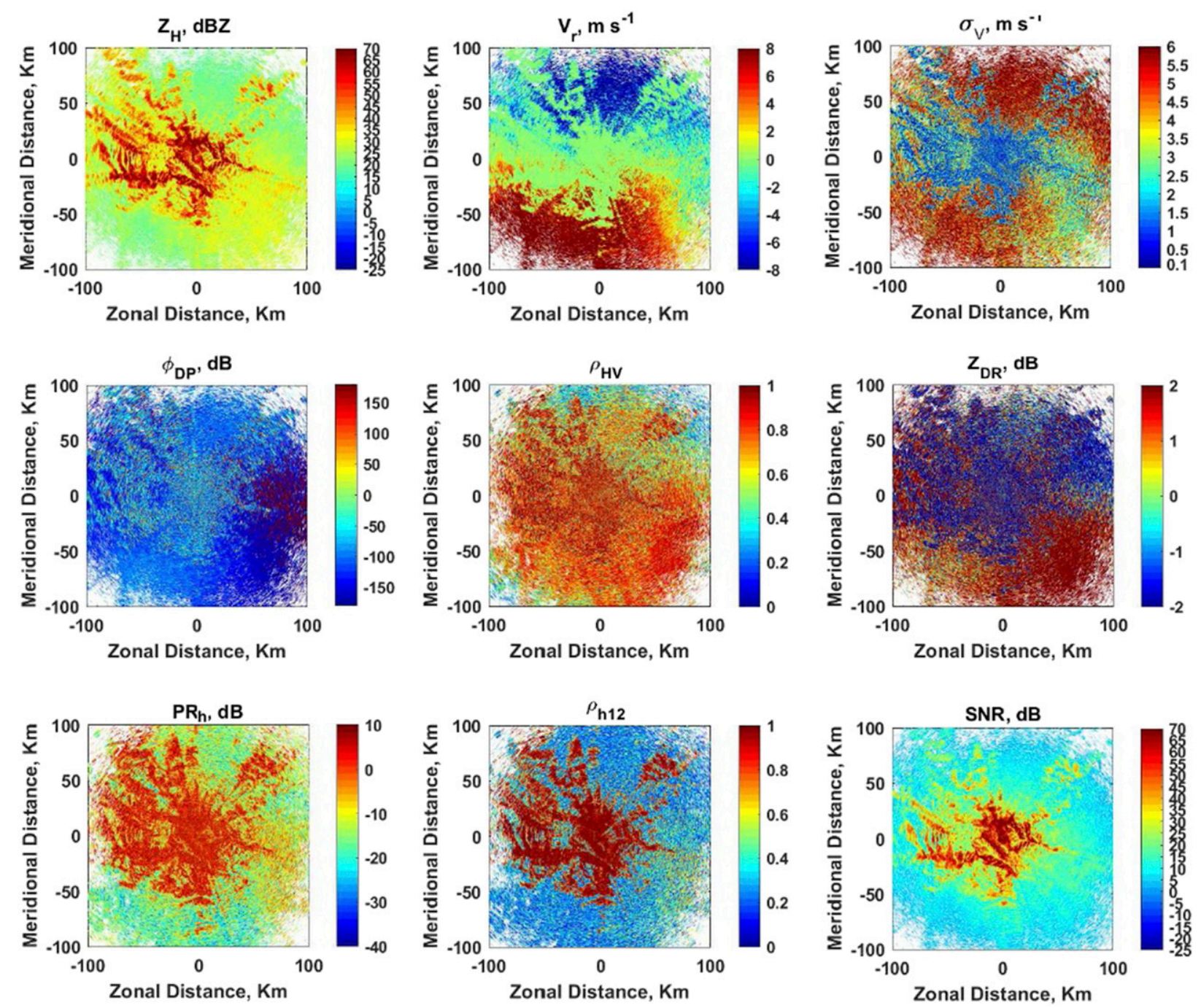

FIG. 1. Radar clutter data collected at 0709 UTC 27 May 2010: (top left) reflectivity, (top center) Doppler velocity, (top right) spectrum width, (middle left) differential phase, (middle center) dual-polarization cross-correlation coefficient, (middle right) differential reflectivity, (bottom left) power ratio, (bottom center) dual-scan cross-correlation coefficient, and (bottom right) SNR.

(i.e., $0.5^{\circ}$ ) under cold, clear-air conditions. The pure weather data collected at ranges beyond ground clutter (generally have negligible clutter) is collapsed to near ranges, and can be combined with the specially collected and edited clutter PPI without range collapsing (GolbonHaghighi et al. 2018; Li et al. 2013). We use other weather and clutter datasets (testing datasets) to evaluate the performance of the detection algorithm. Therefore, the PDFs of discriminant functions are obtained from training datasets for clutter and weather signals, and then the performance is evaluated and compared by applying the PDFs to the testing datasets. The training and testing weather datasets were collected by the KOUN WSR$88 \mathrm{D}$ in snow conditions, and at $1.5^{\circ}$ elevation angle at 1308 and 1402 UTC 9 February 2011, with two scans for each time. The clutter datasets were collected in clearair conditions at low elevation angle (i.e., $0.5^{\circ}$ ), at 0709 UTC 27 May 2010 and at 0046 UTC 4 February 2011. Figures 1 and 2 show reflectivity, Doppler velocity, spectrum width, differential phase, dual-polarization cross-correlation coefficient $\rho_{\mathrm{hv}}$, differential reflectivity $Z_{\mathrm{DR}}$, power ratio (PR), dual-scan cross-correlation coefficient $\rho_{12}$, and SNR for the clutter data collected at 0709 UTC 27 May 2010, and the weather data collected at 1308 UTC 9 February 2011. To avoid noise effects, I/Q data for resolution volumes with SNR less than $20 \mathrm{~dB}$ are subsequently discarded, and to provide a pure clutter and weather fields, data in resolution volumes contaminated by moving point objects (e.g., birds and aircraft) are ignored by deleting the resolution volumes 

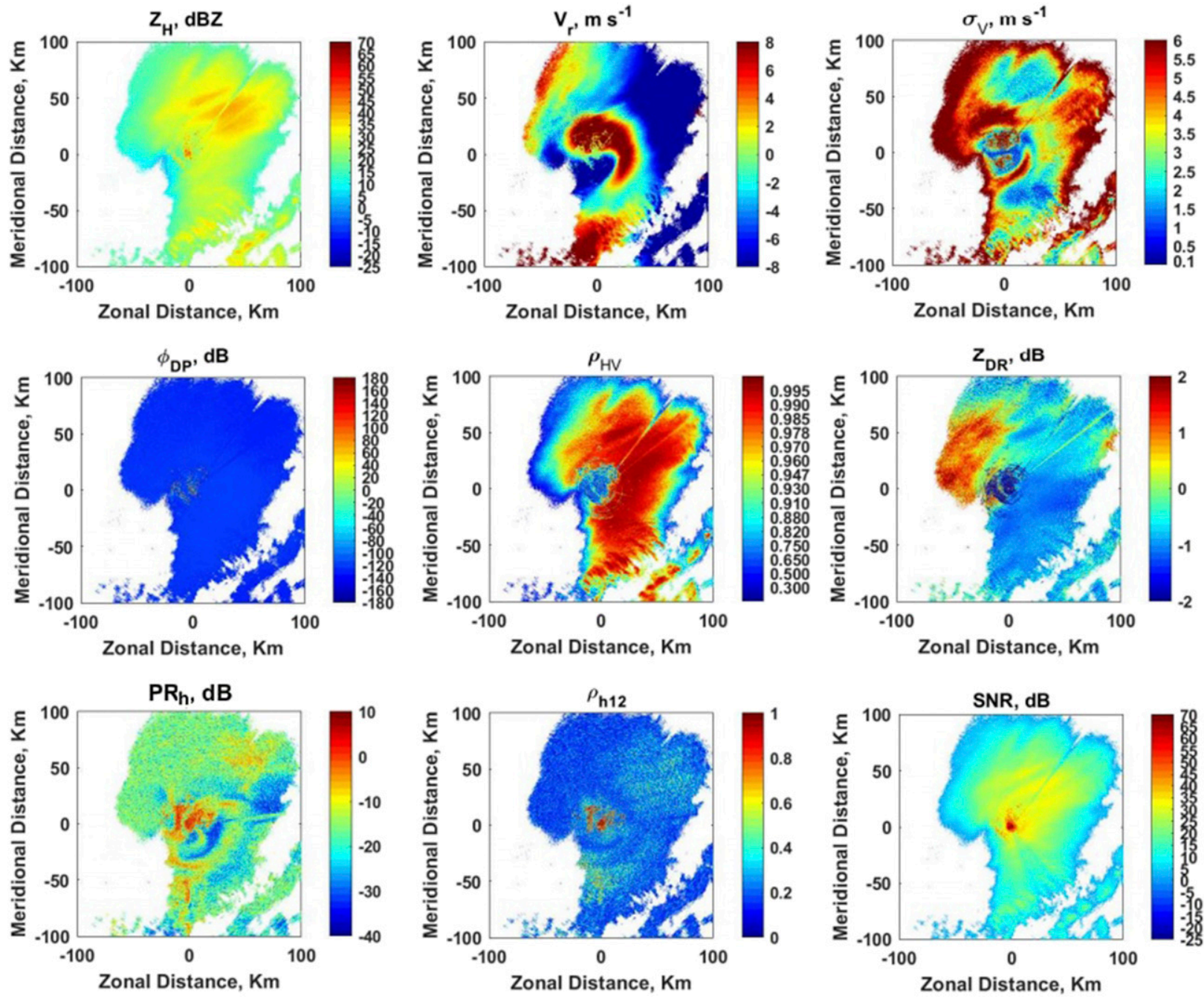

FIG. 2. Radar weather data collected at 1308 UTC 9 Feb 2011: (top left) reflectivity, (top center) Doppler velocity, (top right) spectrum width, (middle left) differential phase, (middle center) dual-polarization cross-correlation coefficient, (middle right) differential reflectivity, (bottom left) power ratio, (bottom center) dual-scan cross-correlation coefficient, and (bottom right) SNR.

showing mean Doppler velocities $\left|v_{r}\right|>1 \mathrm{~ms}^{-1}$ and clutter-to-noise ratio less than $3 \mathrm{~dB}$. The dual scan crosscorrelation coefficient and the phase structure function for $\mathrm{h}$ and $\mathrm{v}$ polarizations are introduced as discriminant functions for detection algorithms in the next subsections.

To obtain the cross-correlation coefficient between two scans, two sequential azimuthal scans with different pulse repetition times (PRTs) are collected by the WSR88D. The short-PRT data (e.g., Ts $2=0.973 \mathrm{~ms}$ ) are downsampled in order to be matched with its corresponding long-PRT data (e.g., Ts1 $=3.10 \mathrm{~ms}$ ) (Golbon-Haghighi et al. 2016).

\section{a. Dual-scan cross-correlation coefficient}

The dual-scan cross-correlation coefficient between two time series' I/Q data, from the same location is defined as (Zhang 2016; Lei et al. 2012; Melnikov and Zrnić 2007)

$$
\begin{aligned}
& \left|\frac{1}{M} \sum_{m=1}^{M} V_{\mathrm{h} 1}(m) V_{\mathrm{h} 2}^{*}(m)\right| \\
& \rho_{12 \mathrm{~h}}=\frac{\sqrt{\left|\frac{1}{M} \sum_{m=1}^{M} V_{\mathrm{h} 1}(m) V_{\mathrm{h} 1}^{*}(m)\right|\left|\frac{1}{M} \sum_{m=1}^{M} V_{\mathrm{h} 2}(m) V_{\mathrm{h} 2}^{*}(m)\right|}}{,} \\
& \rho_{12 \mathrm{v}}=\frac{\left|\frac{1}{M} \sum_{m=1}^{M} V_{\mathrm{v} 1}(m) V_{\mathrm{v} 2}^{*}(m)\right|}{\sqrt{\left|\frac{1}{M} \sum_{m=1}^{M} V_{\mathrm{v} 1}(m) V_{\mathrm{v} 1}^{*}(m)\right|\left|\frac{1}{M} \sum_{m=1}^{M} V_{\mathrm{v} 2}(m) V_{\mathrm{v} 2}^{*}(m)\right|}},
\end{aligned}
$$


where $V$ represents the received complex echo signal sample for the first or second scan; $m$ signifies the echo sample number, and $M$ is the number of samples in dwell time (i.e., the time used to make estimates of polarimetric variables and spectral moments). The dual scan cross-correlation coefficients for h polarization $\rho_{12 \mathrm{~h}}$ and v polarization $\rho_{12 \mathrm{v}}$ have mostly the same PDFs, and we used $\rho_{12}$ as the average of $\rho_{12 \mathrm{~h}}$ and $\rho_{12 \mathrm{v}}$ (Zhang 2016; Li et al. 2014). As normally expected, the PDF of $\rho_{12}$ is larger for clutter than that for weather signals, because the signals received from ground clutter have a longer correlation time than weather signals (Ice et al. 2009; Li et al. 2013).

Narrow-band zero velocity weather signals $W_{0}$ are echoes from resolution volumes where the turbulence and the mean wind radial shear is weak, with a mean radial velocity close to zero; thus, these weather signals commonly have a longer correlation time compared to other weather signals (i.e., $W$ ). We considered $W_{0}$ as a separate weather class because their properties are mostly similar to clutter signals, and thus are the most challenging to distinguish from clutter.

Therefore, the following the three classes $\omega_{i}$ of echoes are being considered for the classification:

1) $C$ : Clutter,

2) $W_{0}$ : Narrow-band zero-velocity weather (i.e., $\left|v_{r}\right| \leq$ $2 \mathrm{~m} \mathrm{~s}^{-1}$ and $\sigma_{v} \leq 2 \mathrm{~m} \mathrm{~s}^{-1}$ ),

3) $W$ : Nonzero-velocity weather (i.e., $\left|v_{r}\right|>2 \mathrm{~m} \mathrm{~s}^{-1}$ or $\left.\sigma_{v}>2 \mathrm{~m} \mathrm{~s}^{-1}\right)$

where $\left|v_{r}\right|$ and $\sigma_{v}$ are the mean Doppler velocity and the spectrum width, respectively (Doviak and Zrnic 2006; Hubbert et al. 2009a). Figure 3 shows the classconditional densities $p\left(\rho_{12} \mid \omega_{i}\right)$, for three classes (i.e., $\left.\omega_{i}=\left\{C, W, W_{0}\right\}\right)$, obtained from training datasets. The class label of each resolution volume for the almost pure clutter and pure weather datasets, has been obtained by using the DPDS algorithm (Golbon-Haghighi et al. 2016; $\mathrm{Li}$ et al. 2013). Although the performance of the DPDS algorithm is entirely acceptable for the controlled training datasets (due to the high $P_{D}$ for CSR $>0 \mathrm{~dB}$ ), all PDFs were verified by the proposed PSF algorithm. It can be seen from this figure that there is a small overlapped range between clutter and weather PDFs, and the peaks of the clutter and weather PDFs are in different ranges.

\section{b. Phase structure function}

The phase structure function is introduced as a novel discriminant function to improve clutter detection algorithms. The wave scattering from the randomly distributed hydrometers yields a rapidly fluctuating phase due to the random size and location of scatterers and turbulence with radial velocity (Figs. 4a,b). However,

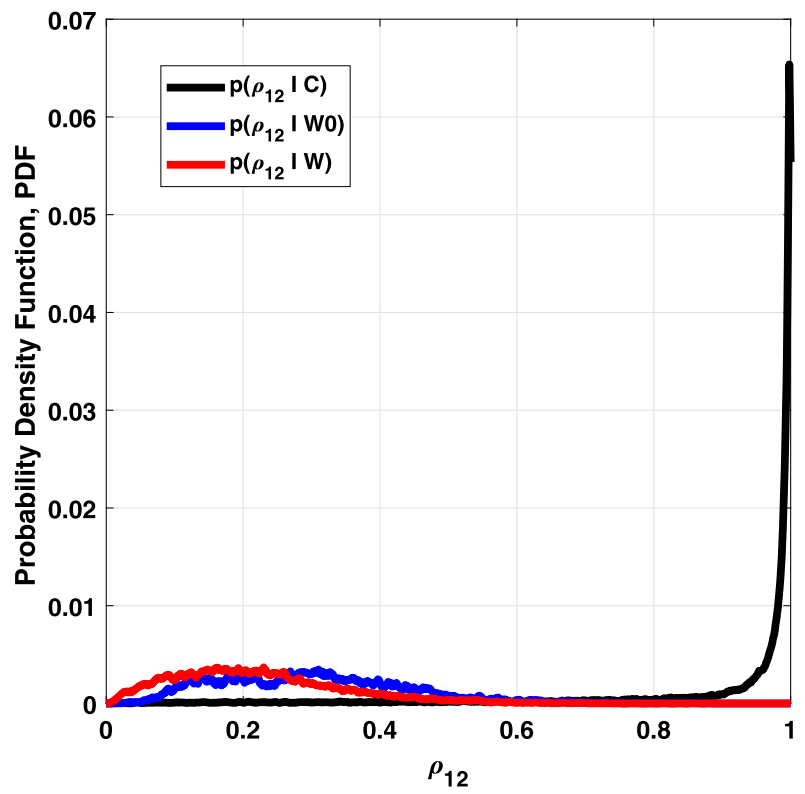

FIG. 3. Dual-scan cross-correlation coefficient $\rho_{12}$.

the wave scattering from fixed scatterers on the ground (i.e., ground clutter) produces a slow fluctuation in the phase of receiving signals (Fig. 4c), due to the scanning beam. As can be seen from Fig. 4, the phase fluctuations for weather signals are stronger than that for ground clutter. Therefore, we introduce the phase structure function to distinguish clutter from weather signals. In Fig. 4, the azimuth is equivalent to the sample time, because the radar antenna is rotating azimuthally with a constant elevation angle. The I/Q data for each resolution volume is represented by a complex voltage sample, and the phase $\phi(\mathrm{rad})$ can be calculated as

$$
\phi=\measuredangle V, \text { where } V=X-j Y=A e^{-j \phi} .
$$

We define the phase structure function (PSF) for $\mathrm{h}$ and $\mathrm{v}$ polarizations as

$$
\begin{gathered}
\operatorname{PSF}_{\mathrm{h}}=\frac{1}{M} \sum_{m=1}^{M-1}\left|\phi_{h}(m+1)-\phi_{h}(m)\right|^{2}, \\
\operatorname{PSF}_{\mathrm{v}}=\frac{1}{M} \sum_{m=1}^{M-1}\left|\phi_{\mathrm{v}}(m+1)-\phi_{\mathrm{v}}(m)\right|^{2} .
\end{gathered}
$$

The phase structure function is obtained from the average of angular separations between consecutive phases for each resolution volume. Figure 5 shows the joint 2D class-conditional densities for the PSF for $h$ and $\mathrm{v}$ polarizations based on the structure function for clutter and weather signals. Parzen windows have been used to obtain the joint class-conditional densities for 

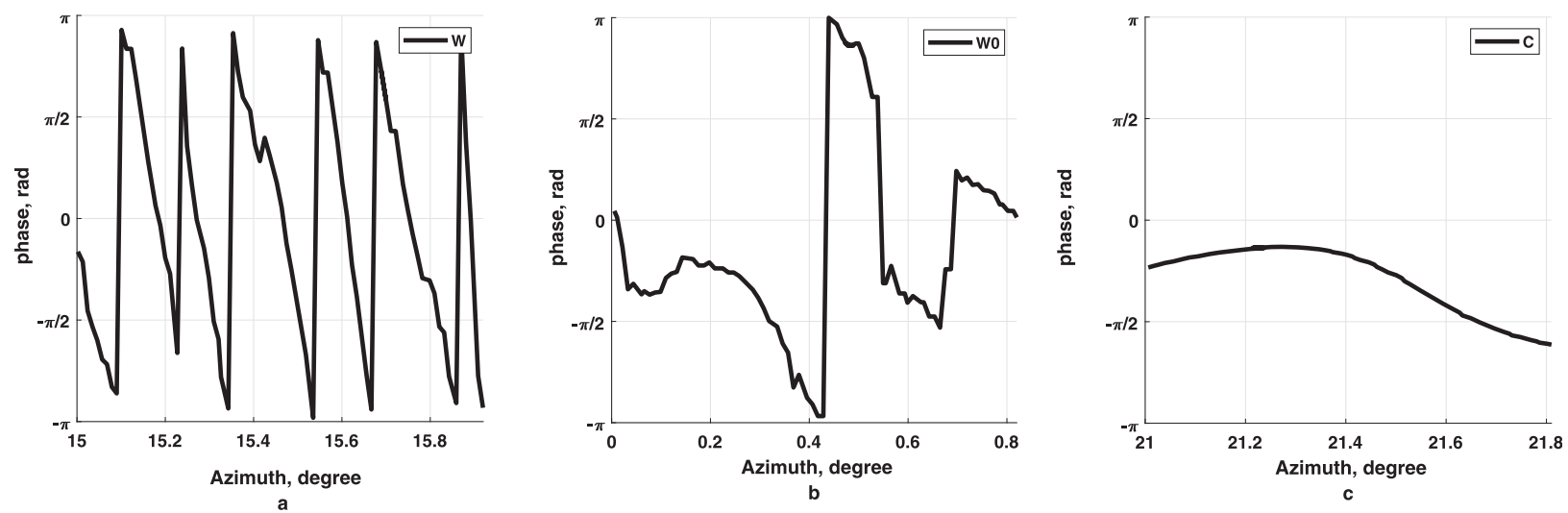

FIG. 4. Phase of the I/Q data for (a) nonzero-velocity weather $W$, (b) zero-velocity weather $W_{0}$, and (c) clutter $C$ signals.

each class [i.e., $p\left(\mathrm{PSF}_{\mathrm{h}}, \mathrm{PSF}_{\mathrm{v}} \mid C\right), p\left(\mathrm{PSF}_{\mathrm{h}}, \mathrm{PSF}_{\mathrm{v}} \mid W\right)$, $\left.p\left(\mathrm{PSF}_{\mathrm{h}}, \mathrm{PSF}_{\mathrm{v}} \mid W_{0}\right)\right]$ from the training datasets (Duda et al. 2012). It is shown that the clutter PDF has mostly near zero values and weather PDFs have larger values with a peak around 7 for $W$. Therefore, there is a good separation between clutter and weather PDFs, and in the next section we show that the PSF as a discriminant function can improve the probability of detection compared to other existing algorithms. The PDFs of discriminant functions may change based on the weather, types of clutter, antenna rotation and the radar parameters, and thus, may need to be updated based on the current condition (Theodoridis and Koutroumbas 2008; McLachlan and Peel 2004). Therefore, to make the PSF algorithm more efficient to use, a multivariate GMM model is used to parameterize the PDFs of discriminant functions. The GMM parameters are estimated based on the ML-EM method (Theodoridis and Koutroumbas 2008; Duda et al. 2012). Figure 6 shows the GMM model for the joint 2D class-conditional densities of $\left[\mathrm{PSF}_{\mathrm{h}}, \mathrm{PSF}_{\mathrm{v}}\right]$, for all classes.

In Fig. 6, the GMM model is obtained from the 2D lognormal distribution function, and the GMM statistical parameters (i.e., mean and covariance vectors) are estimated based on the ML-EM method for each class. Generally, the bivariate (two-dimensional) Gaussian distribution for a $2 \mathrm{D}$ vector $[X, Y]$ can be calculated as (Theodoridis and Koutroumbas 2008; Duda et al. 2012):

$$
\begin{aligned}
p(X, Y) & =\frac{1}{2 \pi \sigma_{X} \sigma_{Y} \sqrt{1-\rho_{X Y}^{2}}} \exp \left[-\frac{1}{2\left(1-\rho_{X Y}^{2}\right)}\right] \exp \left[\frac{\left(X-\mu_{X}\right)^{2}}{\sigma_{X}^{2}}+\frac{\left(Y-\mu_{Y}\right)^{2}}{\sigma_{Y}^{2}}-\frac{2 \rho_{X Y}\left(X-\mu_{X}\right)\left(Y-\mu_{Y}\right)}{\sigma_{X} \sigma_{Y}}\right], \\
\mu & \triangleq\left(\begin{array}{c}
\mu_{X} \\
\mu_{Y}
\end{array}\right), \text { and } \\
\Sigma & \triangleq\left(\begin{array}{cc}
\sigma_{X}^{2} & \sigma_{X} \sigma_{Y} \rho_{X Y} \\
\sigma_{X} \sigma_{Y} \rho_{X Y} & \sigma_{Y}^{2}
\end{array}\right),
\end{aligned}
$$

where $\mu$ and $\Sigma$ are the mean and covariance, respectively, and $\rho_{X Y}$ is the correlation between $X$ and $Y$. In Fig. 6, $[X, Y]$ vector is considered as $\left[\mathrm{PSF}_{\mathrm{h}}, \mathrm{PSF}_{\mathrm{v}}\right]$, and the estimated parameters for the GMM are presented in Table 1.

There are several advantages of parameterizing the PDF of discriminant functions:

1) it can be efficiently trained in different conditions,

2) the classification parameters can be estimated by a relatively small amount of training data, and

3) the Maximum Likelihood algorithm is used for parameter estimation and the maximum a posteriori
(MAP) estimator can be employed in the case that the a priori distributions are not equal for all classes.

The same parameter estimation has been used to estimate the joint 3D class-conditional densities for discriminant functions [i.e., $p\left(\mathrm{PSF}_{\mathrm{h}}, \mathrm{PSF}_{\mathrm{v}}, \rho_{12} \mid C\right), p\left(\mathrm{PSF}_{\mathrm{h}}\right.$, $\left.\left.\mathrm{PSF}_{\mathrm{v}}, \rho_{12} \mid W\right), p\left(\mathrm{PSF}_{\mathrm{h}}, \mathrm{PSF}_{\mathrm{v}}, \rho_{12} \mid W_{0}\right)\right]$ and the GMM model is shown in Figs. 7 and 8.

The joint 3D PDF of the multivariate Gaussian distribution can be obtained as (Theodoridis and Koutroumbas 2008; Duda et al. 2012) 


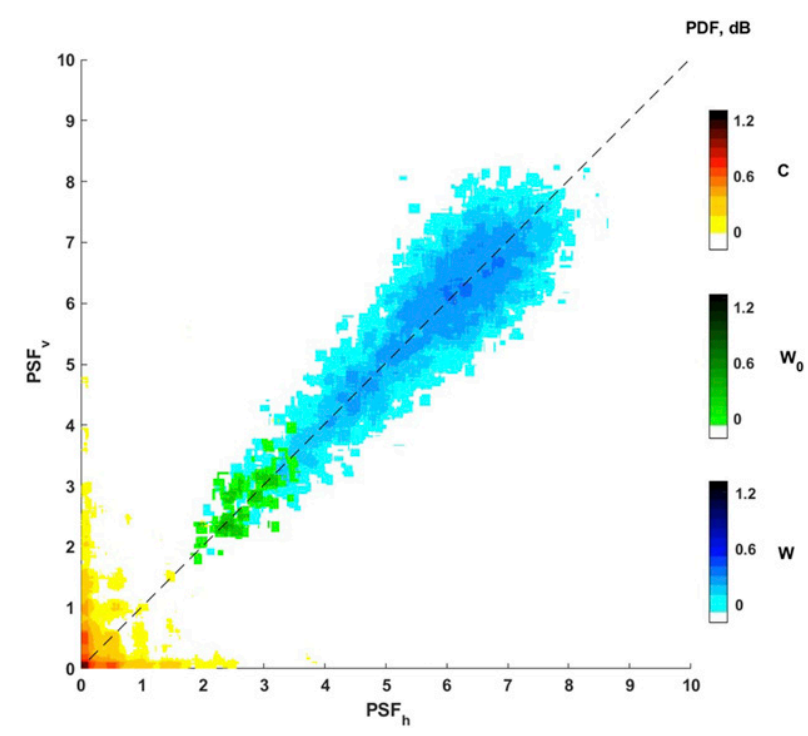

FIG. 5. Joint 2D class-conditional densities for the phase structure function: $p\left(\mathrm{PSF}_{\mathrm{h}}, \mathrm{PSF}_{\mathrm{v}} \mid C\right), p\left(\mathrm{PSF}_{\mathrm{h}}, \mathrm{PSF}_{\mathrm{v}} \mid W\right)$, and $p\left(\mathrm{PSF}_{\mathrm{h}}, \mathrm{PSF}_{\mathrm{v}} \mid W_{0}\right)$.

$$
\begin{aligned}
p(Z) & =\frac{1}{\sqrt{(2 \pi)^{3}|\Sigma|}} \exp \left\{-\frac{1}{2}\left(Z-\mu_{Z}\right)^{\prime} \Sigma^{-1}\left(Z-\mu_{Z}\right)\right\}, \\
\mu & \triangleq\left(\begin{array}{l}
\mu_{Z_{1}} \\
\mu_{Z_{2}} \\
\mu_{Z_{3}}
\end{array}\right), \text { and } \\
\Sigma & \triangleq\left(\begin{array}{ccc}
\sigma_{Z_{1}}^{2} & \sigma_{Z_{1}} \sigma_{Z_{2}} \rho_{Z_{1} Z_{2}} & \sigma_{Z_{1}} \sigma_{Z_{3}} \rho_{Z_{1} Z_{3}} \\
\sigma_{Z_{1}} \sigma_{Z_{2}} \rho_{Z_{1} Z_{2}} & \sigma_{Z_{2}}^{2} & \sigma_{Z_{2}} \sigma_{Z_{3}} \rho_{Z_{2} Z_{3}} \\
\sigma_{Z_{1}} \sigma_{Z_{3}} \rho_{Z_{1} Z_{3}} & \sigma_{Z_{2}} \sigma_{Z_{3}} \rho_{Z_{2} Z_{3}} & \sigma_{Z_{3}}^{2}
\end{array}\right),
\end{aligned}
$$

where $\mathbf{Z}=\left[Z_{1}, Z_{2}, Z_{3}\right]^{\mathrm{T}}=\left[\mathrm{PSF}_{\mathrm{h}}, \mathrm{PSF}_{\mathrm{v}}, \rho_{12}\right]^{\mathrm{T}}$. The fitted parameters of the 3D PDF for the GMM model are presented in Table 2. As normally expected, the weather signals have smaller $\rho_{12}$ with larger PSF values.

Additionally, the clutter has smaller PSF values with a wider range and larger values of $\rho_{12}$. Thus, it is shown that the joint 3D PDF of clutter and weather signals are separated in terms of the proposed 3D discriminant function. Other visualizations of the class-conditional densities are shown in Figs. 8 and 9. Therefore, the PSF detection algorithm based on the 3D PDF can efficiently reduce the error rate and improve the probability of detection compared to other detection algorithms. Also, it can be seen from Figs. 6 and 9 that the joint 2D PDFs have overlapped areas between clutter and weather PDFs and thus, it is normally expected that the error rate

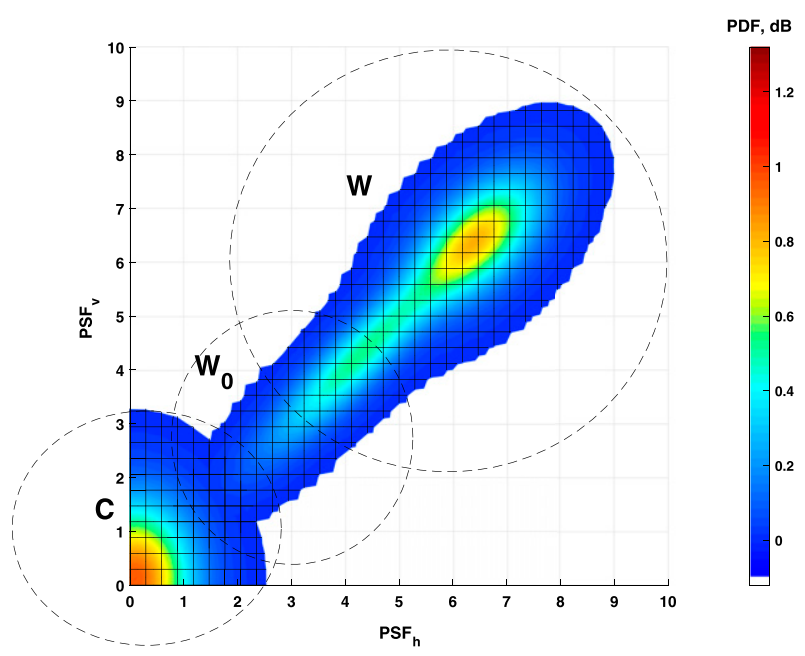

FIG. 6. Gaussian mixture model for the joint 2D classconditional densities: $p\left(\mathrm{PSF}_{\mathrm{h}}, \mathrm{PSF}_{\mathrm{v}} \mid C\right), p\left(\mathrm{PSF}_{\mathrm{h}}, \mathrm{PSF}_{\mathrm{v}} \mid W\right)$, and $p\left(\mathrm{PSF}_{\mathrm{h}}, \mathrm{PSF}_{\mathrm{v}} \mid W_{0}\right)$.

for the PSF algorithm based on the joint 2D PDF is more than that for the 3D PDF. Figure 10 shows the joint 2D class-conditional densities based on $\left[\rho_{12}, \min \left(\mathrm{PSF}_{\mathrm{h}}\right.\right.$, $\left.\left.\operatorname{PSF}_{\mathrm{v}}\right)\right]$ and $\left[\rho_{12}, \mathrm{PSF}_{\mathrm{h}}\right]$. There is a tendency for $\mathrm{PSF}_{\mathrm{h}}$ and $\mathrm{PSF}_{\mathrm{v}}$ to take on values very close to zero (see Fig. 5). Therefore, it is more efficient to use $\min \left(\mathrm{PSF}_{\mathrm{h}}, \mathrm{PSF}_{\mathrm{v}}\right)$ with $\rho_{12}$, for the joint 2D discriminant function, instead of using $\mathrm{PSF}_{\mathrm{h}}$ with $\rho_{12}$. As can be seen from Fig. 10a, the 2D PDF based on $\min \left(\mathrm{PSF}_{\mathrm{h}}, \mathrm{PSF}_{\mathrm{v}}\right)$ can concentrate the $C$ class data nearer the origin while preserving the separation with $W_{0}$ and $W$ classes. In the next section, the probability of detection for our proposed PSF clutter detection algorithm is presented and compared to other detection algorithms.

\section{Bayes optimal decision}

The joint 3D PDF as a discriminant function has been calculated from training datasets, in the previous section, in order to be used as a reference for the Bayesian classifier.

TABLE 1. Fitted class-conditional density parameters for the 2D GMM.

\begin{tabular}{cccc}
\hline \hline Variables & Class & PSF $_{\mathrm{h}}$ & PSF $_{\mathrm{v}}$ \\
\hline$\mu$ & $C$ & 0.1151 & 0.1151 \\
& $W$ & 6.4778 & 6.4286 \\
$\Sigma$ & $W_{0}$ & 2.6999 & 2.6242 \\
& $C$ & 0.4973 & 0.0003 \\
& & 0.0003 & 0.8807 \\
& $W$ & 0.5744 & 0.3126 \\
& & 0.3126 & 0.5816 \\
& $W_{0}$ & 0.3615 & 0.2940 \\
& & 0.2940 & 0.3359 \\
\hline
\end{tabular}




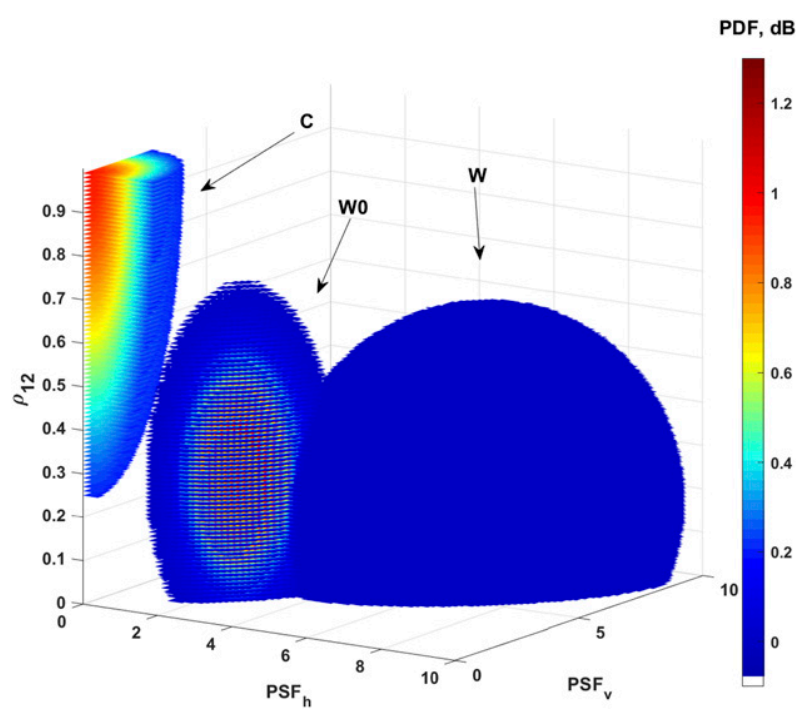

FIG. 7. GMM model for the 3D class-conditional densities: $p\left(\mathrm{PSF}_{\mathrm{h}}\right.$, $\left.\mathrm{PSF}_{\mathrm{v}}, \rho_{12} \mid C\right), p\left(\mathrm{PSF}_{\mathrm{h}}, \mathrm{PSF}_{\mathrm{v}}, \rho_{12} \mid W\right)$, and $p\left(\mathrm{PSF}_{\mathrm{h}}, \mathrm{PSF}_{\mathrm{v}}, \rho_{12} \mid W_{0}\right)$.

The observational discriminant vector $\mathbf{X}^{o}=\left(\mathrm{PSF}_{\mathrm{h}}^{o}, \mathrm{PSF}_{\mathrm{v}}^{o}\right.$, $\left.\rho_{12}^{o}\right)$ is calculated from Eqs. (1) and (2) for each resolution volume. Given an observation of the discriminants $\mathbf{X}^{o}$, the classifier detects $X=\mathbf{X}^{o}$ belongs to the class $\omega_{i}$, if and only if the 3D PDF for $\omega_{i}$ is more than that for other classes. Therefore, $X=\mathbf{X}^{o}$ belongs to the $C$ (or clutter) if and only if

$p\left(\omega_{i} \mid \mathbf{X}^{o}\right)>p\left(\omega_{j} \mid \mathbf{X}^{o}\right), \quad$ for $\quad i, j \in\left[C, W, W_{0}\right], j \neq i$.

According to the Bayes rule, the probability of the $i$ th class given the observed $\mathbf{X}^{o}$ can be obtained as (Miller 1966)

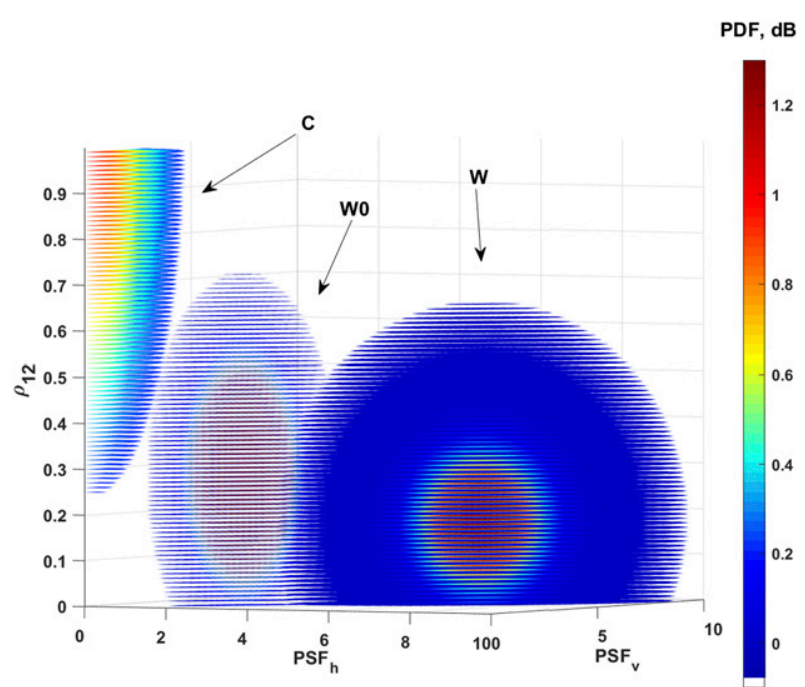

FIG. 8. Other visualizations for the GMM model.
TABLE 2. Fitted class-conditional density parameters for the 3D GMM.

\begin{tabular}{ccccr}
\hline \hline Variables & Class & $\rho_{12}$ & $\mathrm{PSF}_{\mathrm{h}}$ & \multicolumn{1}{c}{$\mathrm{PSF}_{v}$} \\
\hline$\mu$ & $C$ & 0.9815 & 0.1500 & 0.1500 \\
& $W$ & 0.1867 & 6.4744 & 6.4420 \\
& $W_{0}$ & 0.2981 & 2.6460 & 2.5763 \\
$\Sigma$ & $C$ & 0.1872 & 0.0001 & 0.0010 \\
& & 0.0001 & 1.1592 & 0.0003 \\
& & 0.0010 & 0.0003 & 1.5172 \\
& $W$ & 0.0080 & -0.0080 & 0.0009 \\
& & -0.0008 & 0.4414 & 0.2917 \\
& & 0.0009 & 0.2917 & 0.4579 \\
& & 0.0139 & 0.0017 & -0.0010 \\
& & 0.0017 & 0.2062 & 0.1592 \\
& & -0.0010 & 0.1592 & 0.1730 \\
\hline
\end{tabular}

$$
p\left(\omega_{i} \mid X=\mathbf{X}^{o}\right)=\frac{p\left(X=\mathbf{X}^{o} \mid \omega_{i}\right) p\left(\omega_{i}\right)}{p\left(X=\mathbf{X}^{o}\right)} .
$$

It is assumed that the a priori probabilities $p\left(\omega_{\mathrm{i}}\right)$ are equal for all classes, that is, $p(C)=p(W)=p\left(W_{0}\right)=1 / 3$. Furthermore, as $p\left(X=\mathbf{X}^{\mathrm{o}}\right)$ is the same for all classes, Eq. (6) can be rewritten as

$$
p\left(\omega_{i} \mid X=\mathbf{X}^{o}\right)=K p\left(X=\mathbf{X}^{o} \mid \omega_{i}\right),
$$

where $K=1 /\left[3 p\left(X=\mathbf{X}^{o}\right)\right]$ is constant for all classes. Therefore, $p\left(\omega_{i} \mid X=\mathbf{X}^{o}\right)$ is proportional to the likelihood function of classes with respect to $X=\mathbf{X}^{o}$, that is, $p\left(X=\mathbf{X}^{o} \mid \omega_{i}\right)$. The likelihood function can be obtained from the 3D PDFs shown in Fig. 7, for each class. Thus, we have

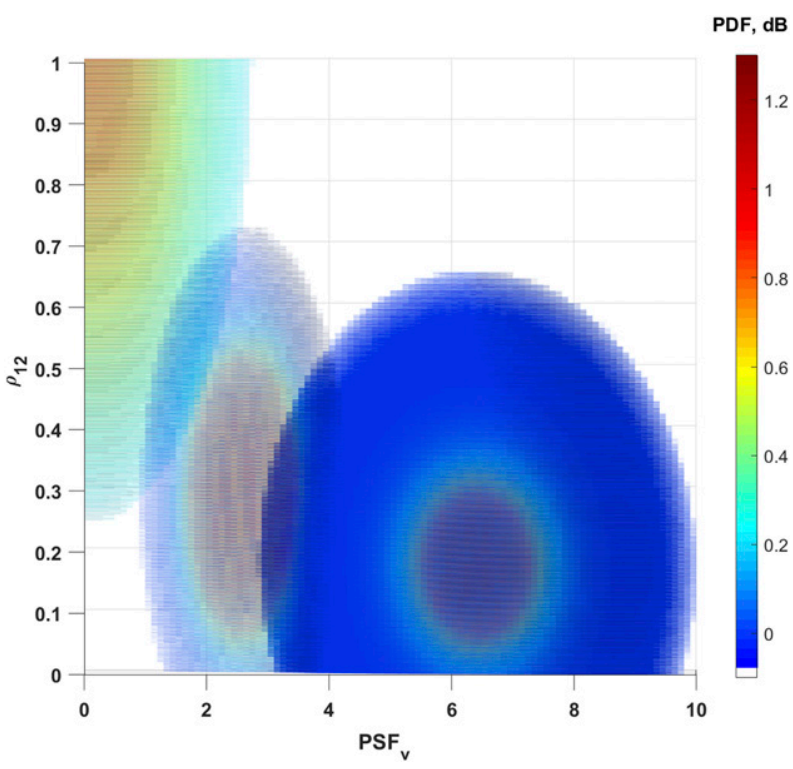

FIG. 9. 2D-visualization of the joint 3D PDF for the GMM model. 

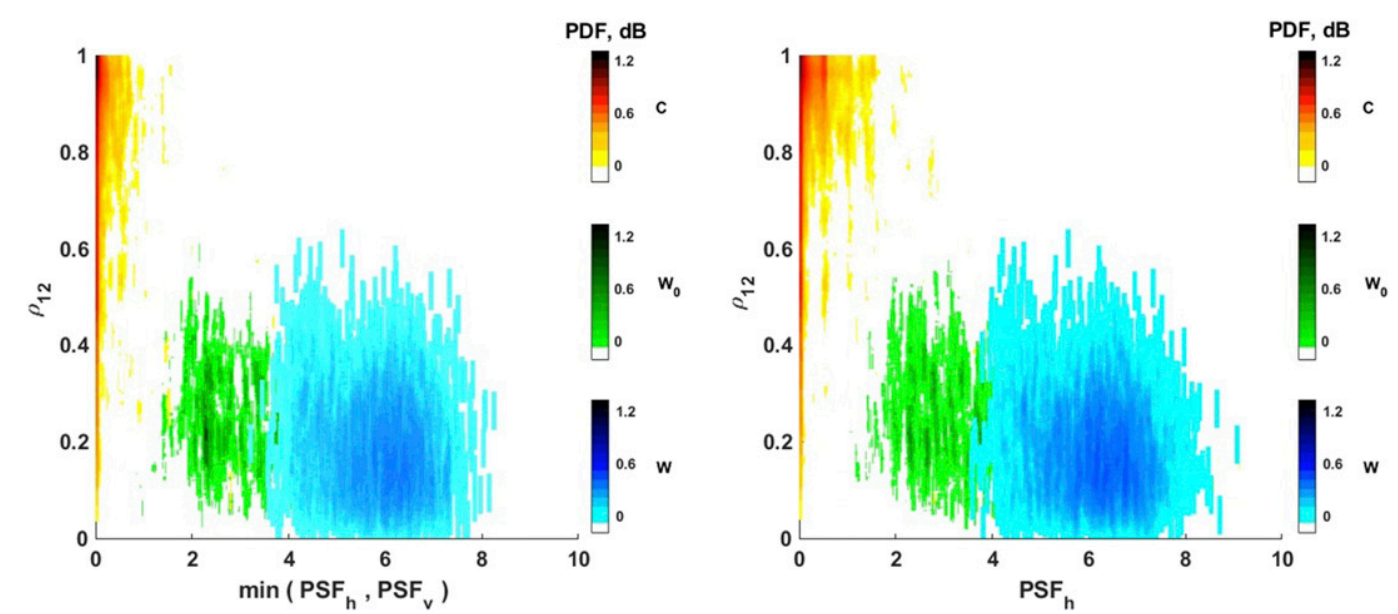

FIG. 10. Joint 2D class-conditional densities: (a) $p\left[\rho_{12}, \min \left(\mathrm{PSF}_{\mathrm{h}}, \mathrm{PSF}_{\mathrm{v}}\right) \mid \omega_{i}\right]$ and (b) $p\left(\rho_{12}, \mathrm{PSF}_{\mathrm{h}} \mid \omega_{i}\right)$.

$$
p\left(X=\mathbf{X}^{o} \mid \omega_{i}\right)=p\left(\operatorname{PSF}_{\mathrm{h}}^{o}, \operatorname{PSF}_{\mathrm{v}}^{o}, \rho_{12}^{o} \mid \omega_{i}\right) .
$$

Therefore, $p\left(X=\mathbf{X}^{o} \mid \omega_{i}\right)$ is calculated for all classes, and the class corresponding to the maximum probability value will be selected as the classification decision. Thus, we can infer that the Bayesian classifier assigns $X=\mathbf{X}^{o}$ to " $C$ " only if

$$
\begin{aligned}
& p\left(\mathrm{PSF}_{\mathrm{h}}^{o}, \mathrm{PSF}_{\mathrm{v}}^{o}, \rho_{12}^{o} \mid C\right)>p\left(\mathrm{PSF}_{\mathrm{h}}^{o}, \mathrm{PSF}_{\mathrm{v}}^{o}, \rho_{12}^{o} \mid W\right) \text { and } \\
& p\left(\mathrm{PSF}_{\mathrm{h}}^{o}, \mathrm{PSF}_{\mathrm{v}}^{o}, \rho_{12}^{o} \mid C\right)>p\left(\mathrm{PSF}_{\mathrm{h}}^{o}, P S F_{\mathrm{v}}^{o}, \rho_{12}^{o} \mid W_{0}\right)
\end{aligned}
$$

The detection algorithm is summarized in the following steps:

1) Calculate the signal-to-noise ratio (SNR) for the current resolution volume. If the SNR is less than $20 \mathrm{~dB}$, the current gate is considered to not have a significant signal compared to noise power, and compute the SNR for the next range resolution volume. Otherwise, go to step 2.

2) Calculate the observed discriminant vector for the current gate, using the 3D PDF shown in Fig. 7:

$$
\begin{gathered}
p\left(X=\mathbf{X}^{o} \mid C\right)=p\left(\mathrm{PSF}_{\mathrm{h}}^{o}, \mathrm{PSF}_{\mathrm{v}}^{o}, \rho_{12}^{o} \mid C\right), \\
p\left(X=\mathbf{X}^{o} \mid W\right)=p\left(\mathrm{PSF}_{h}^{o}, \mathrm{PSF}_{\mathrm{v}}^{o}, \rho_{12}^{o} \mid W\right), \quad \text { and } \\
p\left(X=\mathbf{X}^{o} \mid W_{0}\right)=p\left(\mathrm{PSF}_{\mathrm{h}}^{o}, \mathrm{PSF}_{\mathrm{v}}^{o}, \rho_{12}^{o} \mid W_{0}\right),
\end{gathered}
$$

3) The data for the current gate is clutter contaminated if

$$
p\left(X=\mathbf{X}^{o} \mid C\right)>p\left(X=\mathbf{X}^{o} \mid W\right) \text { and }
$$

$$
p\left(X=\mathbf{X}^{o} \mid C\right)>p\left(X=\mathbf{X}^{o} \mid W_{0}\right) .
$$

Thus, a ground clutter filter (Golbon-Haghighi and Zhang 2019) needs to be applied to mitigate clutter effects and restore weather estimates. Otherwise, the data are not contaminated and return to step 1 for the next gate.

The performance of the clutter detection algorithm is evaluated using the testing control datasets collected by the KOUN WSR-88D at 1402 UTC 9 February and 0047 UTC 4 February 2011. The same procedure has been used to obtain the ground truth from the combination of pure clutter and pure weather data for the testing dataset. Therefore, the ground truth will be used as a reference to obtain the CSR and compare the performance of detection algorithms by changing the CSR value. Our proposed algorithm is compared to other clutter detection algorithms, such as DPDS, DP, DS, and CMD, presented in Golbon-Haghighi et al. (2016), Li et al. (2013, 2014), and Hubbert et al. (2009b).

The probability of detection $P_{D}$ and the probability of false alarm $P_{\mathrm{FA}}$ is defined as

$$
P_{D}=\frac{T P}{T P+F N} \quad \text { and } \quad P_{\mathrm{FA}}=\frac{F P}{F P+T N},
$$

where $T$ and $F$ stand for true and false clutters detected by the algorithm, and $P$ and $N$ stand for the clutter decision made by the algorithm. The probability of false alarm for the controlled testing datasets are presented in

TABLE 3. $P_{\mathrm{FA}}(\%)$ for the testing dataset by using the DPDS, DP, DS, and CMD algorithms.

\begin{tabular}{ccccccccc}
\hline \hline & 3D PSF & 3D PSF GMM & 2D PSF GMM & 1D PSF GMM & DPDS & DP & DS & CMD \\
\hline$P_{\text {FA }}$ & 0.09 & 0.14 & 0.14 & 0.17 & 0.21 & 0.47 & 0.39 & 0.53 \\
\hline
\end{tabular}




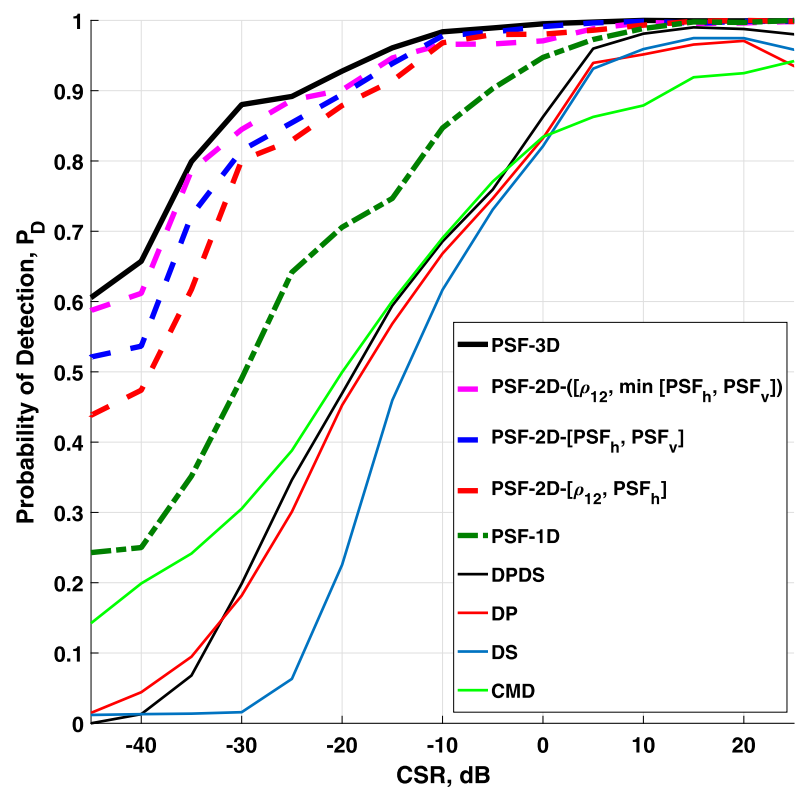

FIG. 11. $P_{D}$ vs CSR compared to other detection methods.

Table 3. It is shown that the proposed 3D PSF clutter detection algorithm produces the lowest $P_{\mathrm{FA}}$ compared to other clutter detection algorithms.

Figure 11 illustrates the probability of detection $P_{D}$ versus CSR for the PSF, DPDS, DP, DS, and CMD algorithms. It is clear that the $P_{D}$ rate should increase with increasing the clutter power or CSR. As shown in Fig. 11, there is a significant improvement in $P_{D}$, using the proposed PSF clutter detection algorithm compared to other existing detection algorithms. The performance improvement for the PSF algorithm is due to the good separation between the clutter and weather PDFs, as shown in Figs. 5-8. Figure 11 also compared the proposed PSF clutter detection algorithm to the PSF based on the joint 2D PDFs, and 3-1D PDF (dashed lines). The joint 2D PDFs have overlapped areas between clutter and weather PDFs (see Figs. 6 and 9) and therefore, the probability of detection for the PSF algorithms based on the joint 2D PDFs are less than that of the joint 3D PDF. The PSF algorithm based on the joint $2 \mathrm{D} \mathrm{PDF}$ of $\left[\min \left(\mathrm{PSF}_{\mathrm{h}}\right.\right.$, $\left.\left.\mathrm{PSF}_{\mathrm{v}}\right), \rho_{12}\right]$, as discriminant function, has a better performance in compared to other 2D PDF discriminant functions, and the PSF algorithm based on the joint 2D PDF of [ $\left.\left(\mathrm{PSF}_{\mathrm{h}}, \mathrm{PSF}_{\mathrm{v}}\right)\right]$ can be used in the case that dual-scan data (or $\rho_{12}$ parameter) is not available for the radar system. Also, the results are compared to the case of 3-1D (nonjoint PDFs of discriminant functions), in which the PDFs are separately calculated and considered to make a decision based on the Bayesian classifier.

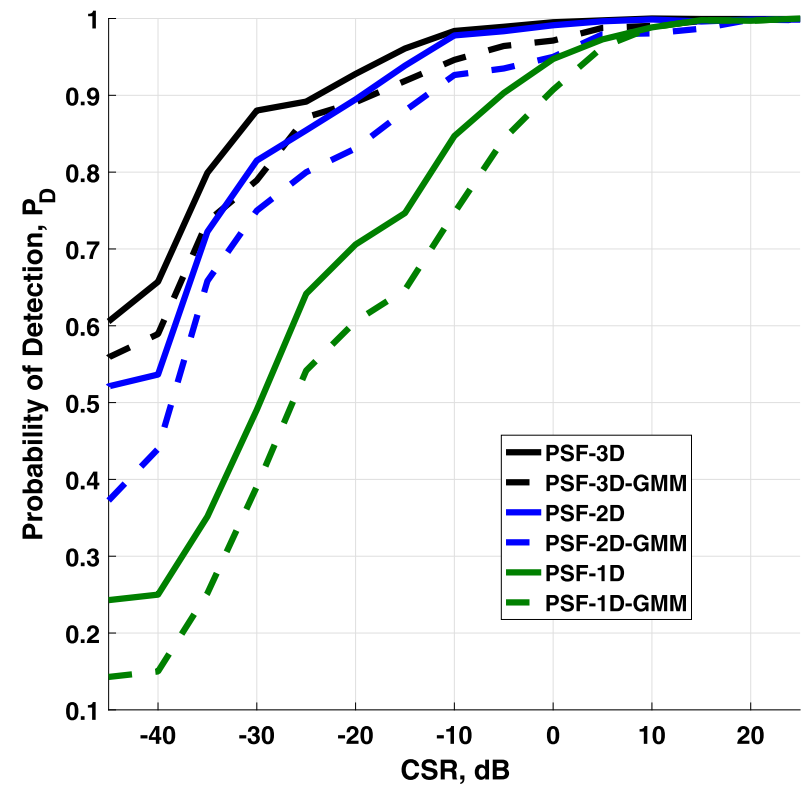

FIG. 12. $P_{D}$ for the PSF clutter detection algorithms compared to PSF-GMM detection algorithms.

As can be seen from Fig. 12, the probability of clutter detection based on the 3D discriminant function has an outstanding performance because there is a good separation between the clutter and weather PDFs, as shown in Fig. 7. The probability of clutter detection for the proposed PSF algorithm is also compared to the PSF algorithms based on the GMM model (dashed lines). It should be noted that the GMM model reduced the computational complexity by parameterizing the PDF of discriminant functions. Although the probability of detection for the GMM is less than that for the PSF, the GMM still has a better performance compared to other existing clutter detection algorithms, such as DPDS, DP, DS, and CMD (Golbon-Haghighi et al. 2016; Li et al. 2013, 2014; Hubbert et al. 2009a). Moreover, the PSF detection algorithms based on the 2D or 1D PDFs still have a better performance compared to other existing detection algorithms, and can be used in case the dual-polarization and/or dual-scan datasets are not available for the weather radar. Figure 13 shows the clutter maps for the proposed PSF algorithm and compared to other clutter detection algorithms. The results of the PSF 3D algorithm are most similar to the ground truth in comparison to other detection algorithms. In this figure, blue points show "true positive" samples and represent the number of clutter samples (as shown in the ground truth) which are correctly detected by the algorithm. Yellow points (false negative) are the samples that are falsely detected as weather instead of clutter by the detection algorithm, and green points (false positive) are 

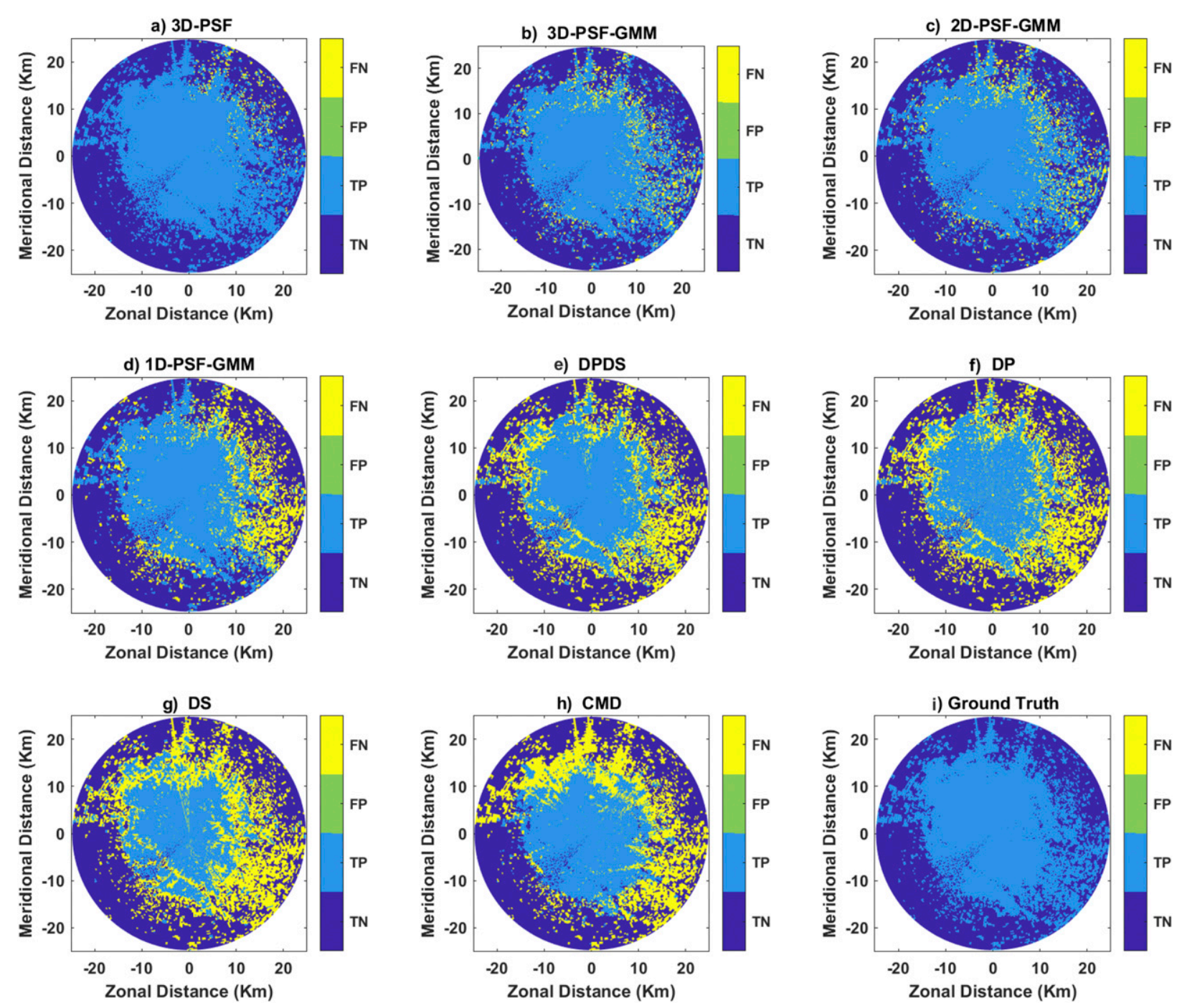

FIG. 13. Clutter maps using (a) 3D PSF algorithm, (b) 3D PSF GMM algorithm, (c) 2D PSF GMM algorithm (PSF , PSF $_{\mathrm{v}}$ ), (d) $1 \mathrm{D}$ PSF GMM algorithm $\left(\mathrm{PSF}_{\mathrm{h}}\right)$, (e) DPDS algorithm, (f) DP algorithm, (g) DS algorithm, (h) CMD algorithm, and (i) ground truth.

the samples that are falsely detected as clutter instead of weather, by the algorithm.

\section{Conclusions}

In this paper, we have proposed a $3 \mathrm{D}$ discriminant function to improve the performance of clutter detection algorithms for weather radars. The phase structure function was introduced as an exceptional discriminant function for clutter detection algorithms. It was shown that this discriminant function had a very good separation between clutter and weather PDFs, even for low CSR values. The PSF has been jointly combined with the dualscan cross-correlation coefficient to form a unique 3D discriminant function. A multivariate Gaussian mixture model was introduced to parameterize clutter and weather PDFs. The parameters of GMM model were estimated based on the ML-EM method. A simple Bayesian classifier was defined to make an optimal decision for the proposed clutter detection algorithm. Practical datasets collected by the KOUN polarimetric WSR-88D were utilized to verify the performance improvement of the proposed detection algorithm compared to other detection algorithms. The KOUN data represents surrounding terrain including clutter from urban, wooded, and prairie areas. The statistical properties of the PSF might be applied to improve clutterfiltering algorithms. Also, the PSF algorithm can be generalized to improve target detection algorithms. The performance of the PSF algorithm to detect clutter from 
heavily foliage woods under different wind conditions should be addressed, in future works.

Acknowledgments. The helpful comments and suggestions provided by the editor and reviewers are greatly appreciated. This work was supported by the National Oceanic and Atmospheric Administration under Grants NA11OAR4320072 and NA16OAR4320115.

\section{REFERENCES}

Bachmann, S. M., 2008: Phase-based clutter identification in spectra of weather radar signals. IEEE Geosci. Remote Sens. Lett., 5, 487-491, https://doi.org/10.1109/LGRS.2008.922733.

Cao, Q., G. Zhang, R. D. Palmer, M. Knight, R. May, and R. J. Stafford, 2012: Spectrum-time estimation and processing (STEP) for improving weather radar data quality. IEEE Trans. Geosci. Remote Sens., 50, 4670-4683, https://doi.org/ 10.1109/TGRS.2012.2190608.

Doviak, R. J., and D. S. Zrnić, 2006: Doppler Radar Weather Observations. 2nd ed. Dover, 592 pp.

Duda, R. O., P. E. Hart, and D. G. Stork, 2012: Pattern Classification. John Wiley \& Sons, 688 pp.

Falconi, M. T., M. Montopoli, and F. S. Marzano, 2016: Bayesian statistical analysis of ground-clutter for the relative calibration of dual polarization weather radars. Eur. J. Remote Sens., 49, 933-953, https://doi.org/10.5721/EuJRS20164949.

Golbon-Haghighi, M.-H., and G. Zhang, 2019: Dual polarization ground clutter filtering. 35th Conf. on Environmental Information Processing Technologies, Phoenix, AZ, Amer. Meteor. Soc., 833, https://ams.confex.com/ams/2019Annual/webprogram/ Paper350230.html.

_,- Y. Li, and R. J. Doviak, 2016: Detection of ground clutter from weather radar using a dual-polarization and dual-scan method. Atmosphere, 7, 83, https://doi.org/10.3390/ atmos7060083.

,-- , and R. J. Doviak, 2018: Ground clutter detection for weather radar using phase fluctuation index. IEEE Trans. Geosci. Remote Sens., 57, 2889-2895, https://doi.org/10.1109/ TGRS.2018.2878378.

Gourley, J. J., P. Tabary, and J. P. du Chatelet, 2007: A fuzzy logic algorithm for the separation of precipitating from nonprecipitating echoes using polarimetric radar observations. J. Atmos. Oceanic Technol., 24, 1439-1451, https://doi.org/ 10.1175/JTECH2035.1.

Groginsky, H. L., and K. M. Glover, 1980: Weather radar canceller design. Preprints, 19th Conf. on Radar Meteorology, Miami Beach, FL, Amer. Meteor. Soc., 192-198.

Hubbert, J. C., M. Dixon, S. M. Ellis, and G. Meymaris, 2009a: Weather radar ground clutter. Part I: Identification, modeling, and simulation. J. Atmos. Oceanic Technol., 26, 1165-1180, https://doi.org/10.1175/2009JTECHA1159.1.

$\longrightarrow,-$, and $-2009 \mathrm{~b}$ : Weather radar ground clutter. Part II: Real-time identification and filtering. J. Atmos. Oceanic Technol., 26, 1181-1197, https://doi.org/10.1175/2009JTECHA1160.1.

Ice, R. L., and Coauthors, 2009: Automatic clutter mitigation in the wsr-88d, design, evaluation, and implementation. 34th Conf. on Radar Meteorology, Williamsburg, VA, Amer. Meteor. Soc., P5.3, https://ams.confex.com/ams/34Radar/techprogram/ paper_155409.htm.
Lei, L., G. Zhang, R. J. Doviak, R. Palmer, B. L. Cheong, M. Xue, Q. Cao, and Y. Li, 2012: Multilag correlation estimators for polarimetric radar measurements in the presence of noise. J. Atmos. Oceanic Technol., 29, 772-795, https://doi.org/ 10.1175/JTECH-D-11-00010.1.

Li, Y., G. Zhang, and R. J. Doviak, 2011: A new approach to detect the ground clutter mixed with weather echoes. 2011 IEEE RadarCon (RADAR), Kansas City, MO, IEEE, 622-626, https://doi.org/10.1109/RADAR.2011.5960612.

$\longrightarrow,-,-$, L. Lei, and Q. Cao, 2013: A new approach to detect ground clutter mixed with weather signals. IEEE Trans. Geosci. Remote Sens., 51, 2373-2387, https://doi.org/10.1109/ TGRS.2012.2209658.

,-- , and - , 2014: Ground clutter detection using the statistical properties of signals received with a polarimetric radar. IEEE Trans. Sig. Proc., 62, 597-606, https://doi.org/ 10.1109/TSP.2013.2293118.

McLachlan, G., and D. Peel, 2004: Finite Mixture Models. John Wiley \& Sons, $419 \mathrm{pp}$.

Meischner, P., 2002: Weather Radar Principles and Advanced Applications. Springer-Verlag, 337 pp.

Melnikov, V. M., and D. S. Zrnić, 2007: Autocorrelation and crosscorrelation estimators of polarimetric variables. J. Atmos. Oceanic Technol., 24, 1337-1350, https://doi.org/10.1175/ JTECH2054.1.

Miller, I., 1966: Probability, Random Variables, and Stochastic Processes. Taylor \& Francis, $852 \mathrm{pp}$.

Park, H. S., A. V. Ryzhkov, D. S. Zrnić, and K.-E. Kim, 2009: The hydrometeor classification algorithm for the polarimetric WSR88D: Description and application to an MCS. Wea. Forecasting, 24, 730-748, https://doi.org/10.1175/2008WAF2222205.1.

Rico-Ramirez, M. A., and I. D. Cluckie, 2008: Classification of ground clutter and anomalous propagation using dual-polarization weather radar. IEEE Trans. Geosci. Remote Sens., 46, 1892-1904, https://doi.org/10.1109/ TGRS.2008.916979.

Siggia, A., and R. Passarelli, 2004: Gaussian model adaptive processing (GMAP) for improved ground clutter cancellation and moment calculation. Proc. Third European Conf. on Radar Meteorology, Vol. 2, Gotland, Sweden, ERAD, 67-73.

Steiner, M., and J. A. Smith, 2002: Use of three-dimensional reflectivity structure for automated detection and removal of nonprecipitating echoes in radar data. J. Atmos. Oceanic Technol., 19, 673-686, https://doi.org/10.1175/1520-0426(2002) 019<0673:UOTDRS $>2.0$. CO; 2 .

Theodoridis, S., and K. Koutroumbas, 2008: Pattern Recognition. 4th ed. Academic Press, 984 pp.

Torres, S. M., and D. A. Warde, 2014: Ground clutter mitigation for weather radars using the autocorrelation spectral density. J. Atmos. Oceanic Technol., 31, 2049-2066, https://doi.org/ 10.1175/JTECH-D-13-00117.1.

Warde, D. A., and S. M. Torres, 2014: The autocorrelation spectral density for Doppler-weather-radar signal analysis. IEEE Trans. Geosci. Remote Sens., 52, 508-518, https://doi.org/ 10.1109/TGRS.2013.2241775.

Wen, G., A. Protat, and H. Xiao, 2017: An objective prototype-based method for dual-polarization radar clutter identification. Atmosphere, 8, 72, https://doi.org/ 10.3390/atmos8040072.

Zhang, G., 2016: Weather Radar Polarimetry. CRC Press, 322 pp., https://doi.org/10.1201/9781315374666. 